\title{
The ASY-EOS Experiment at GSI
}

P. Russotto ${ }^{1}$, M. Chartier ${ }^{2}$, M.D. Cozma $^{3}$, E. De Filippo ${ }^{1}$, A. Le Fèvre ${ }^{4}$, S. Gannon ${ }^{2}$, I. Gašparić ${ }^{5,6}$, M. Kišs ${ }^{4,5}$, S. Kupny ${ }^{7}$,

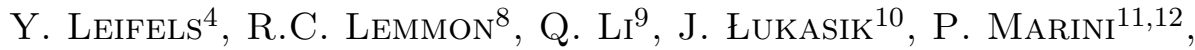
P. Pawlowski ${ }^{10}$, W. Trautmann ${ }^{4}$, L. Acosta $^{13}$, M. Adamczyk $^{7}$, A. Al-Ajlan ${ }^{14}$, M. Al-Garawi ${ }^{15}$, S. Al-Homaidhi ${ }^{14}$, F. Amorini ${ }^{13}$,

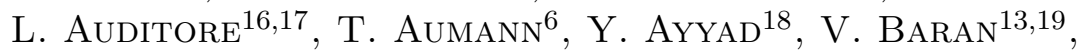
Z. BASRAK ${ }^{5}$, R. BASSINI ${ }^{20}$, J. Benlliure ${ }^{18}$, C. BoiAno ${ }^{20}$, M. Boisjoli ${ }^{12}$, K. BoretzKY ${ }^{4}$, J. BrzYChCZYK ${ }^{7}$, A. Budzanowski ${ }^{10}$, G. Cardella $^{1}$, P. Cammarata $^{21}$, Z. Chajecki $^{22}$, A. Chbihi $^{12}$, M. Colonna ${ }^{13}$, B. Czech $^{10}$, M. Di Toro ${ }^{13,23}$, M. Famiano ${ }^{24}$, V. Greco ${ }^{13,23}$, L. Grassi ${ }^{5}$, C. Guazzoni ${ }^{20,25}$, P. Guazzoni $^{20,26}$, M. Heil ${ }^{4}$, L. Heilborn ${ }^{21}$, R. Introzzi ${ }^{27}$, T. Isobe $^{28}$, K. Kezzar $^{15}$, A. Krasznahorkay ${ }^{29}$, N. Kurz ${ }^{4}$, E. La Guidara ${ }^{1}$,

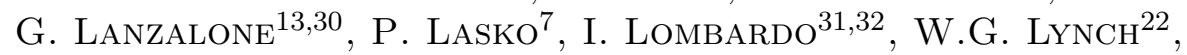
Z. Matthews ${ }^{2}$, L. MaY ${ }^{21}$, T. Minniti ${ }^{1}$, M. Mostazo ${ }^{18}$, A. Pagano ${ }^{1}$, M. Papa ${ }^{1}$, S. Pirrone ${ }^{1}$, R. Pleskac ${ }^{4}$, G. Politi ${ }^{1,23}$, F. Porto ${ }^{13,23}$, R. Reifarth ${ }^{4}$, W. Reisdorf ${ }^{4}$, F. RicCiO ${ }^{20,25}$, F. Rizzo ${ }^{13,23}$, E. Rosato ${ }^{31,32}$, D. Rossi ${ }^{4,22}$, S. SAntoro ${ }^{16,17}$, H. Simon ${ }^{4}$

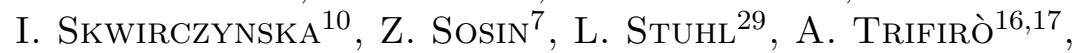

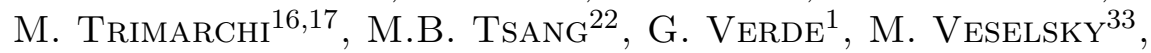
M. Vigilante ${ }^{31,32}$, A. Wieloch ${ }^{7}$, P. WigG ${ }^{2}$, H.H. Wolter ${ }^{34}$,

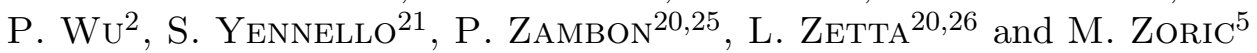


${ }^{1}$ INFN, Sezione di Catania, Catania, Italy

${ }^{2}$ University of Liverpool, Liverpool, UK

${ }^{3}$ IFIN-HH, Măgurele-Bucharest, Romania

${ }^{4}$ GSI Helmholtzzentrum, Darmstadt, Germany

${ }^{5}$ Ruder Boškovic̀ Institute, Zagreb, Croatia

6 Technische Universität, Darmstadt, Germany

7 Jagiellonian University, Krakòw, Poland

8 STFC Laboratory, Daresbury, UK

${ }^{9}$ Huzhou Teachers College, China

${ }^{10}$ IFJ-PAN, Krakòw, Poland

${ }^{11}$ CENBGn Université de Bordeaux, CNRS/IN2P3, Gradignan, France 12 GANIL, Caen, France

13 INFN-Laboratori Nazionali del Sud, Catania, Italy

${ }^{14}$ KACST Riyadh, Riyadh, Saudi Arabia

${ }^{15}$ King Saud University, Riyadh, Saudi Arabia

${ }^{16}$ INFN-Gruppo Collegato di Messina, Messina, Italy

17 Università di Messina, Messina, Italy

18 University of Santiago de Compostela, Santiago de Compostela, Spain

${ }^{19}$ University of Bucharest, Bucharest, Romania

20 INFN-Sezione di Milano, Milano, Italy

${ }^{21}$ Texas A\&M University, College Station, TX, USA

${ }^{22}$ NSCL Michigan State University, East Lansing, MI, USA

${ }^{23}$ Università di Catania, Catania, Italy

${ }^{24}$ Western Michigan University, MI, USA

${ }^{25}$ Politecnico di Milano, Milano, Italy

${ }^{26}$ Università di Milano, Milano, Italy

${ }^{27}$ INFN, Politecnico di Torino, Torino, Italy

${ }^{28}$ RIKEN, Wako, Japan

${ }^{29}$ Institute of Nuclear Research, Debrecen, Hungary

${ }^{30}$ Università Kore, Enna, Italy

31 INFN, Sezione di Napoli, Napoli, Italy

${ }^{32}$ Università di Napoli, Napoli, Italy

${ }^{33}$ Institute of Physics, Slovak Academy of Sciences, Bratislava, Slovakia ${ }^{34}$ LMU, München, Germany

\begin{abstract}
The elliptic-flow ratio of neutrons with respect to protons or light complex particles in reactions of heavy ions at pre-relativistic energies has been proposed as an observable sensitive to the strength of the symmetry term of the nuclear equation of state at supra-saturation densities. In the ASY-EOS experiment at the GSI laboratory, flows of neu-
\end{abstract}


trons and light charged particles were measured for ${ }^{197} \mathrm{Au}+{ }^{197} \mathrm{Au}$ collisions at $400 \mathrm{MeV} /$ nucleon. Flow results obtained for the $\mathrm{Au}+\mathrm{Au}$ system, in comparison with predictions of the UrQMD transport model, confirm the moderately soft to linear density dependence of the symmetry energy deduced from the earlier FOPI-LAND data.

\section{Introduction}

The neutron-proton elliptic flow ratio and difference have been proposed as effective probes of the high-density behavior of the nuclear symmetry energy [1]. The comparison of existing data from the FOPI-LAND experiment [1] with calculations performed with the UrQMD transport model [2] suggests a moderately soft to linear symmetry term. The result suffers, however, from the considerable statistical uncertainty of the experimental data [1]. The same data set was also compared to calculations performed with the Tübingen QMD model and a constraint compatible with the UrQMD result was obtained [3]. At the same time, a thorough study of the parameter dependence of the model predictions was performed in order to devise a route towards a model independent constraint of the the high-density symmetry energy. It was, in particular, found that different parameterizations of the isovector part of the equation of state, the Gogny inspired (momentum dependent) vs. the power law (momentum independent) potential, lead to very similar results. In order to improve the statistical accuracy of the experimental flow parameters for the $\mathrm{Au}+\mathrm{Au}$ reaction and to extend the flow measurements to other systems, the symmetric collision systems ${ }^{197} \mathrm{Au}+{ }^{197} \mathrm{Au},{ }^{96} \mathrm{Zr}+{ }^{96} \mathrm{Zr}$ and ${ }^{96} \mathrm{Ru}+{ }^{96} \mathrm{Ru}$ at $400 \mathrm{MeV} /$ nucleon incident energies have been chosen for the ASY-EOS experiment, conducted at the GSI laboratory in May 2011 (S394 experiment).

\section{Results of the ASY-EOS experiment}

The Large Area Neutron Detector (LAND) [4] was positioned to cover laboratory angles around $45^{\circ}$ with respect to the beam direction, at a distance of about $5 \mathrm{~m}$ from the target. A veto-wall of plastic scintillators in front of LAND allowed discriminating neutrons and charged particles. In this configuration, it was possible to measure the direct and elliptic flows of neutrons and charged particles at mid-rapidity with high precision in the same angular acceptance. In addition, the Krakòw Triple Telescope Array, KRATTA [5], was installed to measure the energy, emission angles and isotopic composition of light charged reaction products. The 35 modules of KRATTA were placed opposite to LAND at a distance of $40 \mathrm{~cm}$ from the target, at polar angles between $24^{\circ}$ and $68^{\circ}$. Very good isotopic resolution 


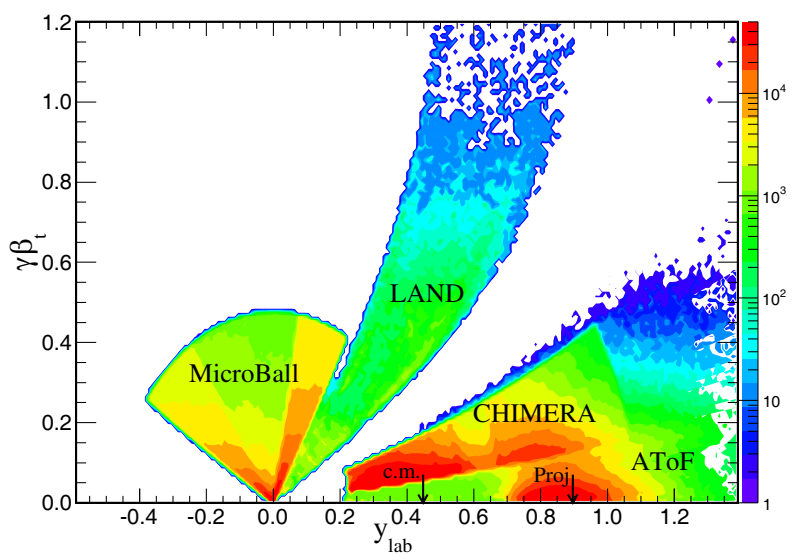

Figure 1: Measured invariant hit distribution for ${ }^{197} A u+{ }^{197} A u$ collisions at 400 $\mathrm{MeV} /$ nucleon incident energy in the transverse-velocity vs. rapidity plane for charged particles detected with the three systems Microball, CHIMERA, and AToF Wall with full azimuthal coverage and for neutrons detected with LAND. The velocity of particles detected with the Microball is not measured and shown here with an assumed distribution.

has been obtained in the whole dynamic range up to $Z \sim 6$. The determination of the impact parameter and the orientation of the reaction plane required the use of several devices: i) the ALADIN Time-of-Flight (AToF) wall [6] was used to detect charged particles at polar angles smaller than $7^{\circ}$; two walls (front and rear) of $2.5^{*} 100 \mathrm{~cm}^{2}$ plastic scintillators, read by two photo-multipliers at both ends, gave information on emission angle, atomic number and velocity of forward-emitted ions; ii) 50 thin (between 3.6 and $5.6 \mathrm{~mm}) \mathrm{CsI}(\mathrm{Tl})$ elements, read out by photo-diodes and arranged in 4 rings, of the Washington-University Microball array [7], surrounded the target at polar angles between $60^{\circ}$ and $147^{\circ}$ with the aim of measuring the multiplicty and angular distribution of backward emitted particles in order to discriminate against background reactions on non-target material; iii) $352 \mathrm{CsI}(\mathrm{Tl})$ scintillators, $12 \mathrm{~cm}$ thick, of the CHIMERA multidetector [8], arranged in 8 rings with $2 \pi$ azimuthal coverage around the beam axis and covering polar angles between $7^{\circ}$ and $20^{\circ}$, were used to detect light charged particles from mid-rapidity emissions.

The kinematic coverage achieved with this assembly of detection systems is illustrated in Fig. 1. In particular, the enhanced particle yields in the kinematic regimes of participant and spectator emissions are clearly visible. The product yields from the decay of the projectile spectator seen with 


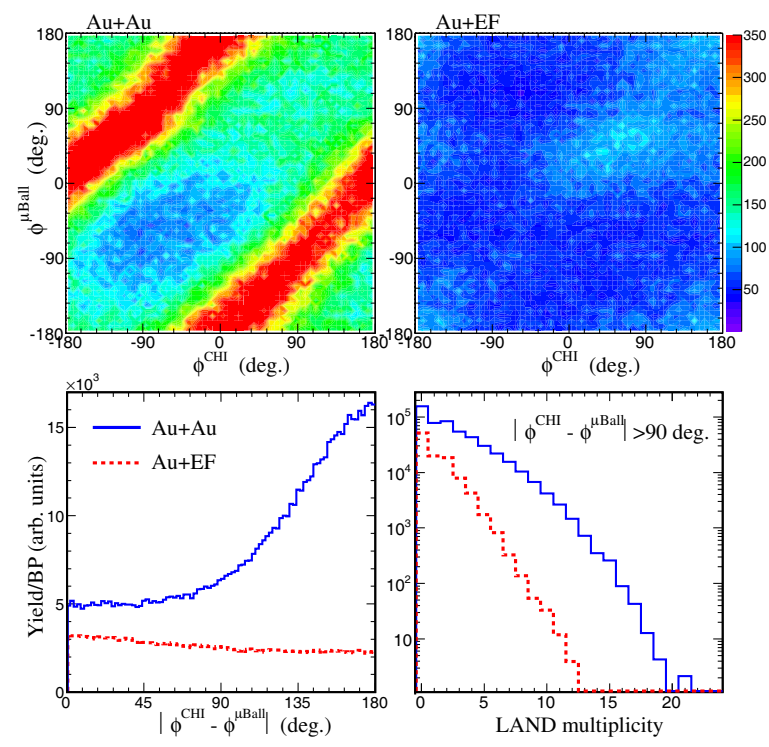

Figure 2: Top row: Correlation between the Q-vector orientations determined with CHIMERA (abscissa) and with the Microball (ordinate) for data sets taken with (left panel) and without (right panel) a target foil in place (EF $=$ Empty Frame). Bottom row: difference of the $\mathrm{Q}$-vector orientations for $\mathrm{Au}+\mathrm{Au}$ and for $\mathrm{Au}+\mathrm{EF}$ data (left panel), normalized with respect to the integrated beam intensity (BP stands for beam particles), and the raw hit multiplicities (right panel) registered with LAND for $\mathrm{Au}+\mathrm{Au}$ (full line) and for $\mathrm{Au}+\mathrm{EF}$ data sets (dotted).

CHIMERA and the AToF Wall do not exactly match because the AToF efficiency for hydrogen isotopes in this energy range is lower than that of the CHIMERA modules.

With beam intensities of about $10^{5}$ pps and targets of $1-2 \%$ interaction probability, about $5 * 10^{6}$ events were collected for each system. Special runs were performed with and without target, in order to measure the background from interaction of projectile ions with air, and with iron shadow bars in front of LAND or the neutron background measurement.

For rejecting background reactions due to the interaction of $\mathrm{Au}$ projectiles with non-target material (mainly air), the correlation of the two reaction plane orientations given by CHIMERA and by the Microball detectors, was used, as shown in fig. 2. From the Microball data, the reaction plane orientation has been estimated by averaging over the azimuthal directions of the recorded hits, i.e. by calculating a $\vec{Q}$ vector (see Ref. [9] for definition) as $\vec{Q}=\sum_{i=1}^{N} \hat{r}_{t}^{i}$, where $\hat{r}_{t}^{i}$ is the azimuthal unit vector in the direction of 
the location of the detector module that recorded the $i^{\text {th }}$ hit and $N \geq 2$. For CHIMERA, the $\vec{Q}$ vector has been calculated as $\vec{Q}=\sum_{i=1}^{N} Z^{i} \beta_{t}^{i} \gamma^{i}$, with $N \geq 4$, taking only the forward emitted particles with rapidity in the center of mass reference frame $y_{i}^{C M}>0.1$. Figure 2 shows the correlation between CHIMERA and Microball reaction plane orientations for $\mathrm{Au}+\mathrm{Au}$ reactions (top left panel) and Au+Empty Frame (top right panel) data, relative to each other according to the integrated beam intensity. In top left panel, the strong anti-correlation for on-target reactions is evident, as expected because CHIMERA covers the forward and the Microball mainly the backward hemispheres in the c.m. frame. In runs with empty target frames, the recorded yields are low and only a weak positive correlation is observed. The difference between the two reaction plane orientations, normalized with respect to the integrated beam intensity, is presented in bottom left panel. In order to minimize the contributions of non-target collisions in the data analysis, an anti-correlation of the CHIMERA and Microball reaction-plane orientations was required. The condition $\left|\Phi_{R P}^{C H I}-\Phi_{R P}^{\mu B a l l}\right|>90^{\circ}$ was applied, resulting in a relative weight of background reactions of less than $20 \%$. This clearly shows the relevance of the Microball data in rejecting background reactions. Bottom right panel of Fig. 2 shows the LAND raw multiplicity (number of fired paddles) normalized with respect to the integrated beam intensity for $\mathrm{Au}+\mathrm{Au}$ and $\mathrm{Au}+$ no target data, after applying the indicated CHIMERA-Microball anticorrelation condition. The contribution from nontarget backgrounds in the kinematic region of LAND is apparently rather weak.

For the impact parameter selection global variables were constructed from the CHIMERA and AToF data. Strong correlations between the used global variables and a good agreement between experimental data and UrQMD calculations was found. The reaction plane orientation has been obtained by merging CHIMERA and AToF data Using the method of [10] we have also estimated the correction factors for the Fourier parameters of the azimuthal distribution, i.e. $v_{1}$ and $v_{2}$ attenuation factors. Azimuthal distributions of neutrons and light charged particles measured with LAND with respect to the reaction plane given by CHIMERA have been extracted for $\mathrm{Au}+\mathrm{Au}$ reactions from runs with and without the shadow bar. After subtracting the background, the obtained distributions have been fitted with formula (1) of Ref. [1] in order to determine the direct $\left(v_{1}\right)$ and elliptic $\left(v_{2}\right)$ flow coefficients. Due to insufficient energy resolution in the LAND veto-wall, charge identification with the $\Delta \mathrm{E}$-vs.-time-of-flight technique has not been possible. Therefore, the results for neutrons and for all recorded charged particles $(\mathrm{Ch})$ are presented. The $v_{1}$ and $v_{2}$ parameters for $\mathrm{Ch}$ and neutrons 


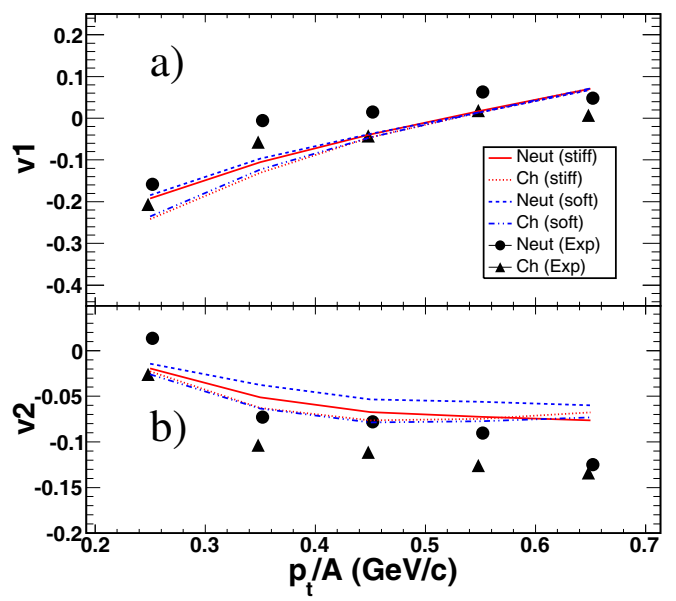

Figure 3: Measured flow parameters $v_{1}$ (top) and $v_{2}$ (bottom) for impact parameter $\mathrm{b}<7.5 \mathrm{fm}$ in $\mathrm{Au}+\mathrm{Au}$ collisions at $400 \mathrm{MeV} /$ nucleon for neutrons (black full dots) and charged particles (black full triangles) as a function of the transverse momentum $p_{t} / A$. The UrQMD predictions for neutrons and charged particles obtained with a stiff $(\gamma=1.5$, red full and dotted lines, respectively) and a soft $(\gamma=0.5$, blue dashed and dash-dotted lines, respectively) density dependence of the symmetry term have been filtered to correspond to the geometrical acceptance of the experiment.

as functions of the transverse momentum $p_{t} / A$ are shown in Fig. 3, respectively. In the same figure, also the UrQMD predictions for a soft $(\gamma=0.5)$ and a stiff $(\gamma=1.5)$ symmetry energy potential [1] are given. Constraints for the symmetry energy were determined as in Ref. [1] by comparing the ratios of the elliptic flows of neutrons and $\mathrm{Ch}, v_{2}^{n} / v_{2}^{C h}$, with the corresponding UrQMD predictions for the soft and stiff cases. The results obtained as functions of the transverse momentum $p_{t} / A$ are shown in Fig. 4. A preliminary value for the power-law coefficient $\gamma$, deduced by linearly interpolating between the predictions, is $\gamma=0.75 \pm 0.10$. The new constraint is compatible with the previous one presented in Ref. [1] by using FOPI-LAND data and the same UrQMD model but the statistical error has been significantly reduced by a factor of $\sim 2$. The determination of systematic uncertainties is still on-going; a preliminary analysis indicates that they are substantial and may lead to an overall final uncertainty of the order of $\Delta \gamma \approx \pm 0.20$. Comparisons of the experimental data with other theoretical transport models will be useful in order to pursue the route towards a model-independent constraint of the high-density symmetry energy initiated in Ref. [3]. The 


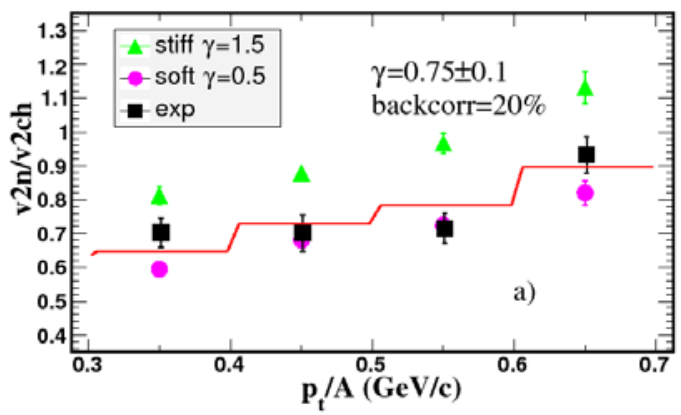

Figure 4: Elliptic flow ratio of neutrons and charged particles for moderately central $(b<7.5 \mathrm{fm})$ collisions as a function of the transverse momentum per nucleon $p_{t} / A$. The full squares represent the experimental data, the triangles and dots represent the UrQMD predictions for stiff $(\gamma=1.5)$ and soft $(\gamma=0.5)$ power-law exponent of the potential term. The full line is the result of a linear interpolation between the predictions, leading to the indicated $\gamma=0.75 \pm 0.10$.

promising preliminary results of the present experiment may also be seen as an encouragement for extending the measurement of neutron and charged particle flows to other reaction systems and energies. Future experiments may thereby benefit from the unique possibilities offered by the NeuLAND detector presently constructed as part of the $R^{3} B$ experimental set-up and from the availability of radioactive ion beams for reaction studies at FAIR.

\section{References}

[1] P. Russotto et al., Phys. Lett. B697 (2011) 471; M.D. Cozma, Phys. Lett. B700 (2011) 139; P. Russotto et al., Eur. Phys. Jour. A50 (2014) 38; Y. Leifels et al., Phys. Rev. Lett. 71 (1993) 963.

[2] Q. Li et al., J. Phys G 31 (2005) 1359.

[3] M.D. Cozma et al., Phys. Rev. C88 (2013) 044912.

[4] Th. Blaich et al., NIM A314 (1992) 136.

[5] J. Łukasik et al., Nucl. Instr. and Meth. A709 (2013) 120.

[6] A. Schüttauf et al., Nucl. Phys. A607 (1996) 457.

[7] D.G. Sarantites et al., Nucl. Instr. and Meth. 381 (1996) 418.

[8] A. Pagano et al., Nucl. Phys. A734 (2004) 504.

[9] P. Danielewicz et al., Phys. Lett. B157 (1985) 146.

[10] J.-Y. Ollitrault, preprint nucl-ex/9711003. 